\title{
Marie MianowsKI, Post-Celtic Tiger Landscapes in Irish Fiction, London, Routledge, 2017
}

\section{Sylvie Mikowski}

\section{OpenEdition}

Journals

Édition électronique

URL : http://journals.openedition.org/etudesirlandaises/5398

DOI : 10.4000/etudesirlandaises.5398

ISSN : 2259-8863

\section{Éditeur}

Presses universitaires de Caen

\section{Édition imprimée}

Date de publication : 29 novembre 2017

Pagination : 159-161

ISBN : 978-2-7535-7388-8

ISSN : 0183-973X

\section{Référence électronique}

Sylvie Mikowski, « Marie MıAnowskı, Post-Celtic Tiger Landscapes in Irish Fiction, London, Routledge, 2017 », Études irlandaises [En ligne], 42-2 | 2017, mis en ligne le 29 novembre 2017, consulté le 24 septembre 2020. URL : http://journals.openedition.org/etudesirlandaises/5398 ; DOI : https://doi.org/ 10.4000/etudesirlandaises.5398

\section{(c) (i) 8 (2)}

Études irlandaises est mise à disposition selon les termes de la Licence Creative Commons Attribution - Pas d'Utilisation Commerciale - Partage dans les Mêmes Conditions 4.0 International. 
de Joyce et son rejet du matérialisme historique au profit d'une approche psychomorphe de l'Histoire. Le chapitre 4 approfondit l'idée d'une celticité joycienne conçue comme un "état inconscient » (p.150) et une porte d'entrée vers l'inquiétante étrangeté. Le chapitre suivant, peut-être plus convaincant, se penche sur l'intérêt de Joyce pour le bricolage et le recyclage interculturels de James Macpherson. Ainsi, selon Barlow, "[i]f Macpherson's work was an effort to construct a memorial for an endangered civilization in the face of modernity and imperialism. Finnegans Wake replicates the attempt to create a spectral "memory", an inner Celtic state as an alternative to materialism" (p. 158). Le chapitre conclusif est quant à lui dévoué à l'empreinte de Robert Burns et envisage enfin l'influence de Joyce sur les poètes et romanciers écossais du vingtième siècle - dont bien sûr Hugh MacDiarmid.

The Celtic Unconscious a le grand mérite de lever le voile sur une part de l'œuvre joycienne jusqu'ici peu explorée. Si le modèle de la "Caledonian antisyzygy " est désormais contesté, il est sollicité avec précaution et à bon escient, systématiquement appuyé sur des exemples concrets tirés des deux romans principalement abordés, Finnegans Wake et Ulysses. L'ouvrage témoigne d'une originalité et d'une rigueur scientifique qui saura satisfaire autant les spécialistes de Joyce que les irlandistes et scotticistes qui trouveront là, à n'en pas douter et probablement dès le paratexte, matière à débat.

Camille MANFredi

Marie Mianowski, Post-Celtic Tiger Landscapes in Irish Fiction, London, Routledge, 2017, 186 p., ISBN 978-1-4724-8798-8, £110.00 (hardcover).

Dans cet ouvrage, Marie Mianowski invite à une lecture de la littérature irlandaise ultra-contemporaine à travers le prisme de l'espace, du lieu et du paysage. Au premier abord, ces thèmes ne surprendront pas quiconque est familier du contexte irlandais tant la terre, sa possession, sa perte et son contrôle ont joué un rôle majeur dans l'histoire politique, sociale et culturelle du pays. Seamus Heaney avait déjà conceptualisé l'importance du lieu dans l'imaginaire collectif irlandais dans son célèbre texte "A Sense of Place ». Pourtant cette approche semble particulièrement appropriée à l'époque envisagée dans l'ouvrage, celle qui suit l'effondrement de l'économie du Tigre celtique, fondée en partie sur la spéculation immobilière. Ainsi les modifications profondes de la société irlandaise se sont trouvées reflétées par les transformations du paysage urbain ou rural, ce que la littérature a enregistré à son tour, à tel point que la fiction irlandaise des années $2010 \mathrm{a}$ pu donner les signes d'une véritable obsession pour les chantiers de construction, les terrains, les friches 
et autres "ghost estates ", ainsi que pour les personnages de promoteurs immobiliers, de préférence véreux et cyniques. Parmi les œuvres étudiées ici, c'est clairement le cas dans The Forgotten Waltz de Anne Enright, The Devil I know de Claire Kilroy, ou encore dans The Spinning Heart de Donal Ryan. La superbe nouvelle de William Trevor, "At Olive Hill », est également replacée dans le contexte de la fièvre immobilière du Tigre celtique. Mais l'ouvrage établit aussi des liens moins évidents entre écriture et paysage dans $A$ Second Life de Dermot Bolger (dans sa version revue par l'auteur), ou dans City of Bohane de Kevin Barry. Deux chapitres entiers sont également consacrés à l'écriture de l'espace chez Colum McCann. M. Mianowski déplie tout au long des pages les possibilités lexicales et sémantiques des mots "place " et "landscape " : à propos de la nouvelle de "At Olivehill " de Trevor, il s'agit par exemple de l'attachement pour le lieu. À propos de The Forgotten Waltz est établi un lien entre désir de posséder un lieu, soif d'enrichissement et sexualité. En ce qui concerne Donal Ryan, il s'agit plutôt de "place " dans le sens de "community ", avec toutes les dérives néfastes que la ruralité irlandaise peut entraîner. Et pour introduire le chapitre consacré à McCann, M. Mianowski écrit : "we will first elucidate the notions of place, displacement, and placelessness. " Beaucoup des œuvres analysées ont d'ailleurs autant un lien direct avec un lieu, au sens de "espace habité ", qu'avec un paysage : il en est ainsi du domaine de Olivehill, du rôle de la propriété et de la spéculation immobilière dans The Forgotten Waltz, dans The Spinning Heart ou dans The Devil I know, mais aussi des maisons qui jouent un rôle central dans certains récits, comme dans The Gathering, ou dans Transatlantic, qui fait d'ailleurs l'objet d'une magnifique analyse. Une autre analyse particulièrement brillante est celle du roman de Kevin Barry City of Bohane. M. Mianowski suggère que ce que Barry parodie ici, c'est l'aspect névrotique du " sense of place " caractérisant Bohane - autre nom pour l'Irlande d'hier et d'aujourd'hui - qui, à travers une fétichisation du lieu, entretient un rapport stérile et paralysant avec son passé, rapport que M. Mianowski décrit comme " la nostalgie de la nostalgie».

L'ouvrage alterne des micro-lectures très pénétrantes avec des réflexions plus abstraites appuyées entre autres sur les concepts de "containment", ou de "boundedness ", inventés par le philosophe australien Jeff Malpas. Marie Mianowski propose non seulement une lecture de certains textes irlandais contemporains, mais une véritable méthode de lecture, qui emprunte ses concepts et ses outils à d'autres domaines que celui de l'analyse littéraire proprement dite, et qui ouvre des perspectives pour l'étude d'autres littératures que celle de l'Irlande.

Toutefois, bien qu'adoptant une méthodologie précise et bien définie, les lectures proposées ne sont jamais systématiques, car l'auteure sait distinguer dans chaque texte ses particularités; de plus, même si le titre de l'ouvrage suggère un ancrage socio-historique, celui de la crise économique datant de 2008, elle n'uti- 
lise jamais le texte comme prétexte à un commentaire sociologique mais sait au contraire exalter les qualités esthétiques des œuvres considérées.

Sylvie Mıкоwsкi

Niall Ó Dochartaig, Katy HaYward and Elizabeth Meehan (eds), Dynamics of Political Change in Ireland: Making and Breaking a Divided Ireland, Milton Park/NewYork, Routledge, 2016, 288 p., ISBN 978-1-1381-9600-1 (hardcover), £110.00, ISBN 978-1-3156-3806-5 (ebook), £27.99.

Cette collection d'articles, publiée sous la co-direction de Niall Ó Dochartaigh, Katy Hayward et Elizabeth Meehan et préfacée par Brendan O'Leary, contient 13 contributions rassemblées en trois chapitres intitulés successivement Contexts, Competition et Complexity. Cette publication est une forme d'hommage à John Coakley dont Brendan O'Leary fait remarquer, à juste titre, la qualité du travail scientifique qu'il a réalisé au cours de sa riche carrière, à Dublin, à Queen's University Belfast mais aussi à l'échelle internationale - Etudes Irlandaises peut d'ailleurs s'enorgueillir d'avoir publié plusieurs de ses articles.

Dès l'introduction, les trois co-directeurs définissent l'objectif ambitieux de l'ouvrage : il s'agit de voir en quoi les interactions entre d'une part, les partis politiques, la société et les idéologies et d'autre part, les structures institutionnelles étatiques et internationales, ont contribué à façonner mais aussi à déconstruire l'espace politique irlandais depuis la partition. Ils affirment aussi que pour répondre à cette question de manière complète, il est impossible d'ignorer les menaces que le résultat du référendum de juin 2016, favorable au Brexit, fait peser sur les fragiles équilibres politiques, sociaux, économiques et culturels qui avaient été trouvés en Irlande depuis 1998. Les "dynamiques du changement politique en Irlande " sont clairement envisagées dans un cadre pluridisciplinaire (histoire, science politique, économie, sociologie...) et pluridimensionnel (dimensions irlandaises, anglo-irlandaises, européennes et mondiales). Naturellement, avec une telle ambition, la question majeure (même si d'autres, comme celle des genres par exemple, sont évoquées par Y. Galligan et M. Hoewer) est celle de l'évolution des nationalismes, des nations et des États, britannique et irlandais.

Le travail accompli dans cet ouvrage est remarquable et doit, à n'en pas douter, faire plaisir à John Coakley. Il pourra y voir très visiblement le signe de sa grande influence scientifique mais c'est pour deux autres raisons principales qu'on imagine sa satisfaction et celle des autres lecteurs de ce livre. Premièrement, les contributions réunies permettent de comprendre la complexité et la multiplicité des formes du 